\title{
EFEITO DA TAXA DE RESFRIAMENTO NA OCORRÊNCIA DE AUSTENITA RETIDA EM UM AÇO PARA ELETRODOS DE SOLDA
}

\author{
Helder Carvalho Ferreira ${ }^{1,2,3}$ \\ Francisco José Martins Boratto ${ }^{4,2}$ \\ Vicente Lopes Tadeu Buono ${ }^{5,2}$
}

\section{Resumo}

A influência da taxa de resfriamento na ocorrência de austenita retida em um aço de baixo teor de carbono ligado ao $\mathrm{Si}$ e $\mathrm{Mn}$, utilizado na fabricação de eletrodos de solda, é medida aqui através da combinação de seccionamento de corpo de prova Jominy com o método de medição de austenita retida através da difração de raios- $X$. Foi observado que a quantidade de austenita retida aumenta de zero, quando a taxa de resfriamento é menor do que $0,8^{\circ} \mathrm{C} / \mathrm{s}$ e a estrutura é majoritariamente ferrítica, para um valor máximo de $6 \%$, no ponto em que a quantidade de bainita também é máxima. Essa informação passa a guiar assim o projeto do ciclo de resfriamento pós-laminação de fio máquina para eletrodos de solda. O conhecimento da proporcionalidade da austenita retida com a presença de fases duras passa a permitir o uso da difração de raios-X como exame liberatório de fio máquina neste tipo de aço, assim como na simplificação de ciclo de tratamento térmico antes da trefilação.

Palavras-chave: Eletrodo; Austenita retida; Taxa de resfriamento.

\section{INFLUENCE OF COOLING RATE ON THE OCORRENCE OF RETAINED AUSTENITE IN A STEEL FOR WELDING ELECTRODES}

\begin{abstract}
The effect of cooling rate on the occurrence of retained austenite in a low carbon Si-Mn steel, used for welding electrodes, is measured here by combining the sectioning of Jominy test samples with the measurement of retained austenite by X-ray diffraction. It was observed that the amount of retained austenite increases from zero, as the cooling rate is less than $0.8^{\circ} \mathrm{C} / \mathrm{s}$, and the structure is mostly ferrite, to a maximum of $6 \%$, where the quantity of bainite is also maximum. This information guides the post-rolling cooling cycle when welding grade is been rolled. The hard phases proportionality with the amount of retained austenite allows, then, the use of X-ray diffraction as an end of the line equipment for quality control, and also permits simplification of heat treatment before wire drawing.
\end{abstract}

Keywords: Electrode; Retained austenite; Cooling rate.

\section{INTRODUÇÃO}

Os aços de baixo teor de carbono utilizados na fabricação de eletrodos de solda do tipo MIG têm normalmente teores de manganês e silício elevados, o que favorece a ocorrência do constituinte MA (martensita-austenita) o qual apresenta dureza elevada em relação à matriz ferrítica. Esta característica de dureza elevada pode ocasionar fratura do arame durante a trefilação, assim como aumento do limite de resistência acima de um nível considerado adequado para operação de solda.
A caracterização e quantificação da fração volumétrica de austenita retida têm sido amplamente exploradas em aços TRIP, onde a presença deste constituinte (I5-20\%) melhora as propriedades de resistência mecânica e ductilidade devido ao fenômeno da transformação induzida por deformação [1]. Porém, no caso de aços para eletrodos, onde a presença deste constituinte se encontra em níveis baixos $(<6 \%)$, são praticamente inexistentes trabalhos técnicos sobre o comportamento da austenita em fio máquina para trefilação.

'Doutorando em Engenharia Metalúrgica, Materiais e de Minas, Universidade Federal de Minas Gerais - UFMG, Belo Horizonte, MG, Brasil. E-mail: helder.ferreira@arcelormittal.com.br

${ }^{2}$ Associação Brasileira de Metalurgia, Materiais e Mineração - ABM, São Paulo, SP, Brasil.

${ }^{3}$ Arcelormittal Monlevade, João Monlevade, MG, Brasil.

${ }^{4}$ Consultor Independente, Belo Horizonte, MG, Brasil.

${ }^{5}$ Escola de Engenharia, Universidade Federal de Minas Gerais - UFMG, Belo Horizonte, MG, Brasil. 
Neste trabalho é avaliada a influência da taxa de resfriamento na ocorrência de austenita retida para amostras deste tipo de aço. Para se conseguir várias taxas de resfriamento foi seccionado um corpo de prova (CP) submetido ao ensaio Jominy [2].

Para a quantificação da austenita retida foi utilizado o método de comparação direta, através da difração de raios-X [3]. Para melhorar a precisão dos cálculos, foram feitas decomposições das radiações $K \alpha_{1}$ e $K \alpha_{2}$, através de modelamento matemático das intensidades de raios- $X$ com o uso da função de Pearson VII e integração algébrica de cada pico [4].

\section{PROCEDIMENTO EXPERIMENTAL}

\section{I Coleta de Amostra}

Foi coletada uma amostra do perfil intermediário $(\phi=30 \mathrm{~mm})$, durante laminação de uma corrida de aço para eletrodo MIG, fabricada na ArcelorMittal Monlevade, cuja composição química é mostrada na Tabela I. Esta amostra foi usinada para as dimensões padronizadas do ensaio Jominy.

O carbono é um dos elementos de maior influência na microestrutura da solda. O manganês promove o refinamento da microestrutura, porém aumenta a estabilidade da austenita devido sua baixa difusividade nesta fase e tendência de segregação na interfase martensita-austenita [5]. Já o silício é o principal desoxidante do metal de solda, não sendo efetivo na formação de uma estrutura refinada.

\subsection{Cálculo da Taxa de Resfriamento}

Para a amostra usinada foi utilizado o procedimento do ensaio Jominy. A taxa de resfriamento $\left({ }^{\circ} \mathrm{C} / \mathrm{s}\right)$ na temperatura de $700^{\circ} \mathrm{C}$, em cada cota, $\mathrm{X}(\mathrm{mm})$, distante da ponta resfriada, pode ser calculada pela Equação I, obtida através de análise de regressão, com dados de taxa de resfriamento no ensaio Jominy previamente publicados $[2,6]$ :

$$
\operatorname{taxa}=872,7569 X^{-1.547197}
$$

Após a realização do ensaio, foram feitos cortes transversais no CP Jominy. A escolha das cotas foi feita de modo a se obter taxas de resfriamento próximas das praticadas industrialmente no processo Stelmor ${ }^{\circledR}$ (resfriamento controlado pós-laminação de fio máquina). Em seguida, as amostras foram lixadas, polidas e preparadas para análise metalográfica e difração de raios-X.

\subsection{Uso da Difração de Raios-X para Quantificação da Austenita Retida}

A difração por raios- $X$ foi realizada em um difratômetro Shimadzu XRD6000, com radiação de cobalto-K $\alpha$ e uso de monocromador. Utilizou-se velocidade do goniômetro de $0,02^{\circ} \mathrm{C} / \mathrm{s}$ com passo de $3 \mathrm{~s}$. Foi escolhida para análise a faixa
$2 \theta$ de 48 a $80^{\circ}$, uma vez que esta faixa contém os picos mais intensos tanto da fase ferrítica quanto austenítica. Desta forma, quatro picos foram analisados: (I I0) $\alpha,(200) \alpha$, (I I I ) y e (200) $\gamma$. Para aço contendo somente as fases ferrita/martensita e austenita, o cálculo da fração volumétrica da austenita pode ser obtido conforme mostrado na Equação 2 [7]:

$$
\mathbf{V}_{\gamma}=\sum \mathbf{l}_{\gamma}^{(\mathrm{hkl})} / \mathbf{R}_{\gamma}^{(\mathrm{hkl})} /\left(\sum \mathbf{I}_{\alpha}^{(\mathrm{hkl})} / \mathbf{R}_{\alpha}^{(\mathrm{hkl})}+\sum \mathbf{I}_{\gamma}^{(\mathrm{hkl})} / \mathbf{R}_{\gamma}^{(\mathrm{hkl})}\right)
$$

Os valores da intensidade teórica normalmente são padronizados para cada tipo de radiação utilizada ou podem ser calculados. Para a radiação usada aqui, estes valores foram calculados, conforme previsto na literatura $[3,7]$ e estão mostrados na Tabela 2.

Para modelamento matemático dos picos ferríticos e austeníticos foi utilizado a equação Pearson VII [4], cuja forma é apresentada na Equação 3:

$$
I(x)=I_{0}\left[1+\frac{K^{2}\left(x-x_{0}\right)^{2}}{M}\right]^{-M}
$$

$\mathrm{Na}$ Equação $3 x$ é o valor do ângulo $2 \theta$ e $I_{\circ}$ a intensidade máxima do pico para o ângulo $x_{0}$. O parâmetro $K$ controla a largura da curva e o parâmetro $M$ a taxa de decaimento das "caudas".

O modelo completo da intensidade calculada, incluindo ambas as radiações $\lambda_{\mathrm{K} \alpha 1}$ (índices ímpares) e $\lambda_{\mathrm{K} \alpha 2}$ (índices pares) para a ferrita e austenita, pode ser expresso pela Equação 4, onde é incluída, também, a contribuição do ruído ("background"), $a+b x$ :

$$
I(x)=I_{(111) \gamma}(x)+I_{(110) \alpha}(x)+I_{(200) \gamma}(x)+I_{(200) \alpha}(x)+(a+b x)
$$

Tabela I. Composição química (\% em peso) da liga utilizada.

\begin{tabular}{lccc}
\hline Classificação & $\% \mathbf{C}$ & \%Mn & \%Si \\
\hline Liga & 0,095 & $\mathrm{I}, 637$ & $\mathrm{I}, 096$ \\
ER70S-6(*) & $0,06-0, \mathrm{I} 5$ & $\mathrm{I}, 40-\mathrm{I}, 85$ & $0,80-\mathrm{I}, \mathrm{I} 5$ \\
\hline
\end{tabular}

(*) Especificação europeia EN440; Especificação americana AWS A5. I..

Tabela 2. Intensidade teórica relativa $(R)$ para radiação de cobalto, onde foi utilizado comprimento de onda $\lambda_{\text {Kal }}$ de I,78892 $\AA$, monocromador de grafite $\left(2 \theta_{m}\right.$ de $\left.30,94^{\circ}\right)$ e parâmetros de rede de 2,8664 e 3,6440 $\AA$ para ferrita e austenita, respectivamente.

\begin{tabular}{ccc}
\hline hkl & $\mathbf{2} \theta$ & $\mathbf{R}$ \\
\hline$(110) \alpha$ & 52,37 & 140,3 \\
$(200) \alpha$ & 77,23 & 19,90 \\
$(I I I) \gamma$ & 50,82 & 103,2 \\
$(200) \gamma$ & 59,40 & 46,60 \\
\hline
\end{tabular}


Sendo:

$$
\begin{aligned}
& I_{(111) \gamma}(x)=I_{1}\left[1+\frac{K_{1}^{2}\left(x-x_{01}\right)^{2}}{M_{1}}\right]^{-M_{1}}+I_{2}\left[1+\frac{K_{2}^{2}\left(x-x_{02}\right)^{2}}{M_{2}}\right]^{-M_{2}} \\
& I_{(110) \alpha}(x)=I_{3}\left[1+\frac{K_{3}^{2}\left(x-x_{03}\right)^{2}}{M_{3}}\right]^{-M_{3}}+I_{4}\left[1+\frac{K_{4}{ }^{2}\left(x-x_{04}\right)^{2}}{M_{4}}\right]^{-M_{4}} \\
& I_{(200) \gamma}(x)=I_{5}\left[1+\frac{K_{5}^{2}\left(x-x_{05}\right)^{2}}{M_{5}}\right]^{-M_{5}}+I_{6}\left[1+\frac{K_{6}{ }^{2}\left(x-x_{06}\right)^{2}}{M_{6}}\right]^{-M_{6}} \\
& I_{(200) \alpha}(x)=I_{7}\left[1+\frac{K_{7}{ }^{2}\left(x-x_{07}\right)^{2}}{M_{7}}\right]^{-M_{7}}+I_{8}\left[1+\frac{K_{8}^{2}\left(x-x_{08}\right)^{2}}{M_{8}}\right]^{-M_{8}}
\end{aligned}
$$

A otimização dos parâmetros e os cálculos para obtenção das intensidades (áreas) dos picos ferríticos e austeníticos foram feitos utilizando regressão não linear pelo método dos mínimos quadrados [8], com a restrição de que para cada pico a posição relativa das componentes $\mathrm{K} \alpha$, e $\mathrm{K} \alpha_{2}$ respeita a equação de Bragg, com $\lambda_{\text {Kal }}=1,78892 \AA$ e $\lambda_{\text {K } \alpha 2}=1,79278 \AA$. As áreas foram calculadas pela integração algébrica da função de Pearson VII [4].

\section{RESULTADOS}

A Figura I ilustra como foi feita a correlação da taxa de resfriamento com o resultado de difração de raios-X. Como exemplo, são mostrados dois difratogramas para dois planos de corte, relativo a duas taxas de resfriamento conforme previsto pela Equação I. Podem ser vistos nos difratogramas dois picos correspondentes à fase ferrítica $(\alpha)$ e dois correspondentes a austenita $(\gamma)$.

\section{I Fração Volumétrica da Austenita Retida}

Foram observados 18 planos de corte ao longo do comprimento do CP Jominy. Para cada plano (correspondente a uma taxa de resfriamento específica) foram realizados 6 difratogramas em posições equidistantes para cálculo da fração volumétrica com grau de significância de $95 \%$. Como exemplo, a Figura 2 mostra o resultado obtido para uma medida referente à cota de $8,77 \mathrm{~mm}$, o que corresponde a uma taxa de $30,3^{\circ} \mathrm{C} / \mathrm{s}$, incluindo as linhas do modelamento matemático com a função Pearson VII. O cálculo da fração volumétrica da austenita é então obtido pelo uso dos parâmetros optimizados da função de Pearson VII, referente à radiação $\lambda_{\text {Kal }}$ (Tabela 3 ), substituídos na Equação 2 com os valores da intensidade teórica informados na Tabela 2. O

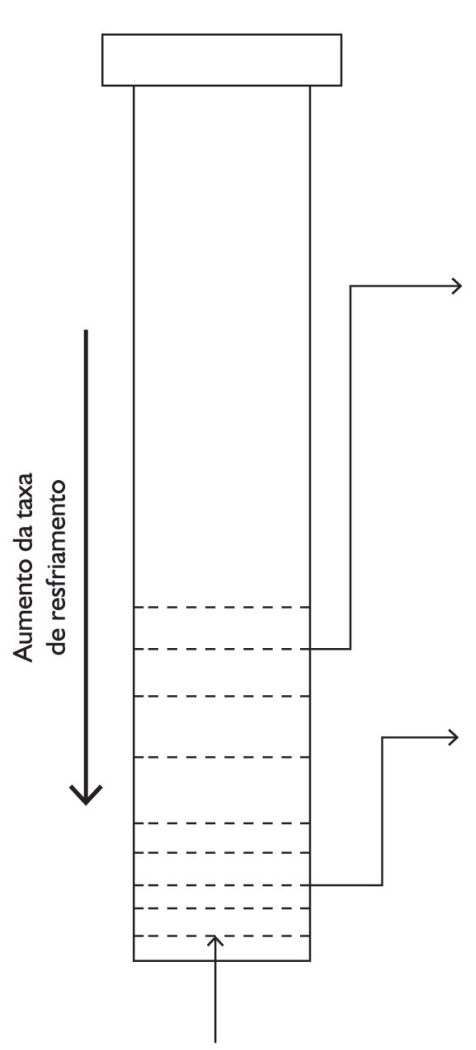

Planos de corte no CP
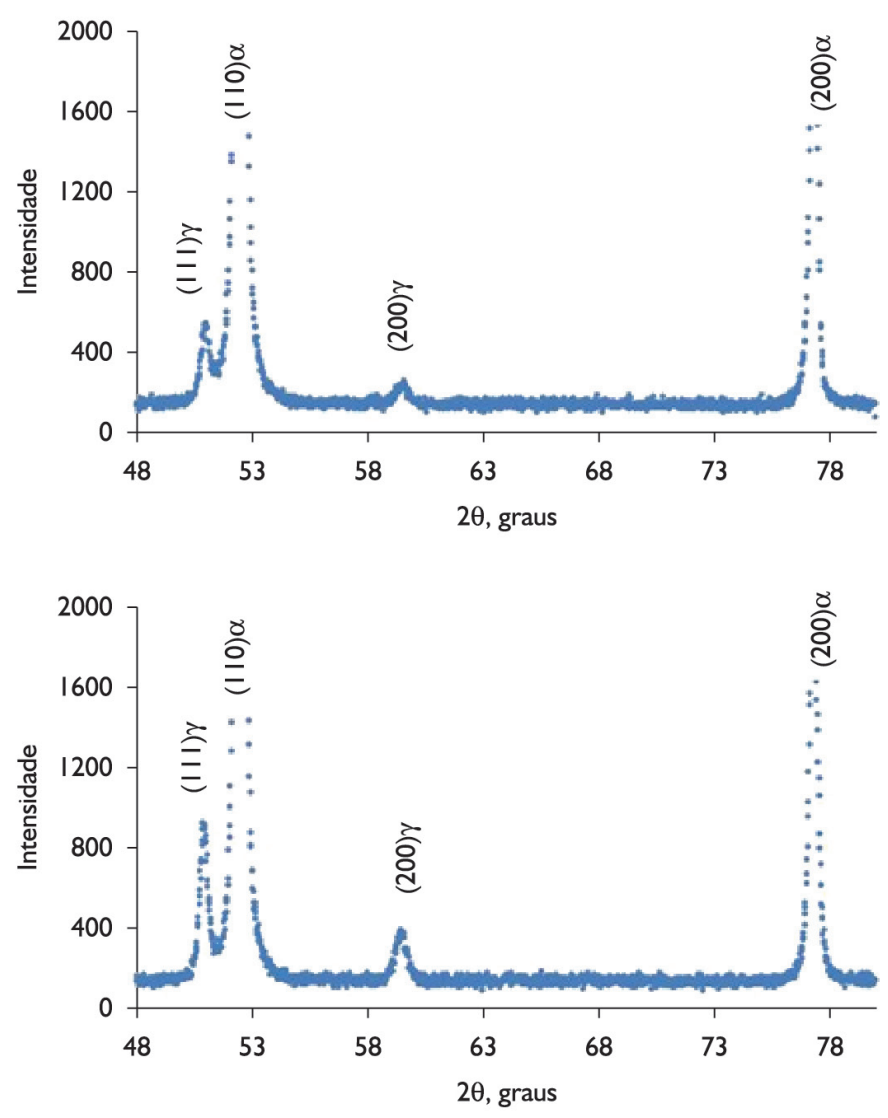

Figura I. Desenho esquemático do corpo de prova (CP) Jominy e os planos de corte realizados para análise na difração. Para cada plano de corte foi realizado difração de raios-X, conforme ilustrado (seis medidas de difração para cada plano). 

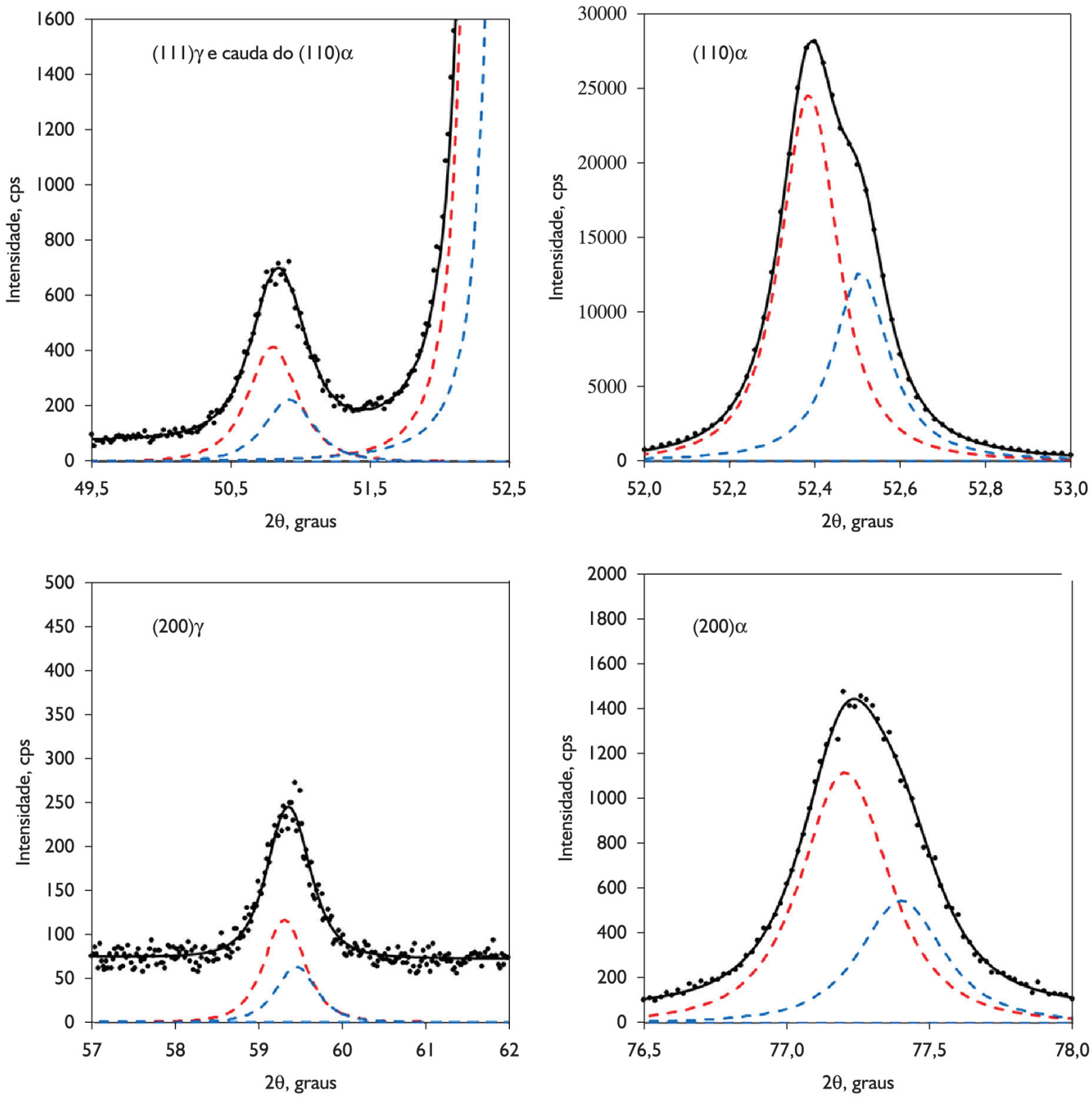

Figura 2. Difratograma da amostra resfriada a uma taxa de $30,3^{\circ} \mathrm{C} / \mathrm{s}$. As linhas pontilhadas correspondem ao comprimento de onda $\lambda_{\mathrm{K} \alpha . \mathrm{l}}$ (pico mais alto) e ao comprimento de onda $\lambda_{\mathrm{K} \alpha 2}$. (pico mais baixo). Os pontos são as medidas experimentais e a linha contínua o resultado do modelamento matemático completo.

Tabela 3. Parâmetros optimizados da função de Pearson VII, pelo método dos mínimos quadrados, correspondente ao difratograma da Figura 2. Comprimento de onda do cobalto $\lambda_{\mathrm{K} \alpha 1}$ e $\lambda_{\mathrm{K} \alpha 2}$ de I,78892 e I,79278 $\AA$, respectivamente. O parâmetro $M$ é adimensional, os outros parâmetros $I_{\mathrm{o}}, x_{\mathrm{o}}, K$ e $I$ têm unidades relacionadas à medição do espectro de difração.

\begin{tabular}{|c|c|c|c|c|c|c|c|c|}
\hline \multirow[t]{2}{*}{ Parâmetro } & \multicolumn{4}{|c|}{$\mathbf{K} \alpha_{1}$} & \multicolumn{4}{|c|}{$\mathbf{K} \alpha_{2}$} \\
\hline & $(I I I) \gamma$ & $(110) \alpha$ & $(200) \gamma$ & $(200) \alpha$ & $(I I I) \gamma$ & $(I I 0) \alpha$ & $(200) \gamma$ & $(200) \alpha$ \\
\hline$K$ (graus $\left.^{-1}\right)$ & 4,47 & 11,95 & 3,20 & 5,14 & 4,47 & 12,67 & 3,20 & 5,43 \\
\hline$x_{0}$ (graus) & 50,80 & 52,39 & 59,31 & 77,20 & 50,92 & 52,51 & 59,45 & 77,40 \\
\hline$M$ & $\mathrm{I}, 70$ & 1,42 & 1,90 & 1,62 & $\mathrm{I}, 70$ & $\mathrm{I}, 17$ & 1,90 & $\mathrm{I}, 46$ \\
\hline$I_{0}(\mathrm{cps})$ & 418 & 24.632 & 117 & 1.120 & 226 & 12.714 & 63 & 547 \\
\hline$I$ (graus.cps) & 218 & 5.177 & 82 & 517 & 118 & 2.795 & 44 & 249 \\
\hline
\end{tabular}


resultado da fração volumétrica de austenita retida para todos os 18 planos de corte está mostrado na Figura 3a. Cada ponto do gráfico corresponde à média das seis medidas. A fração de austenita retida cresce a partir de taxas de resfriamento baixas até aproximadamente $16,6^{\circ} \mathrm{C} / \mathrm{s}$. A partir deste valor, a fração de austenita decresce ligeiramente com o aumento da taxa de resfriamento.

\subsection{Teor de Carbono da Austenita Retida}

A avaliação do teor de carbono $(C \gamma)$ foi feita em função da medida do parâmetro de rede ( $a \gamma$ ) obtido a partir da difração do pico (200)y, de acordo com a Equação 5, onde o efeito do silício e manganês foram considerados desprezíveis em relação ao carbono [9]:

$$
a_{\gamma}=3,4567+0,0467 \mathrm{C}_{\gamma}
$$

Para a medida do parâmetro de rede da austenita foi inicialmente calculado o parâmetro da ferrita para o pico (200) $\alpha$, cujo resultado de 112 mediçóes, referente à radiação $\lambda_{\text {kal }}$, apresentou valor $2 \theta_{\text {kal }}$ de $77,2047^{\circ}$. Desta forma, para cada medição realizada, foi introduzido um fator de correção $\left(f_{2 \theta}\right)$ que é a diferença entre este valor médio e o valor individual de $2 \theta_{\mathrm{k} \alpha \mathrm{l}}$ referente ao pico $(200) \alpha$ (Equação 6):

$$
f_{2 \theta}=77,2047-2 \theta_{\mathrm{kal}}^{(200)}
$$

Desta forma, o parâmetro de rede corrigido da austenita foi calculado através da equação de Bragg para o pico (200)y, e é dado pela Equação 7:

$$
a_{\gamma}=\frac{\lambda}{\operatorname{sen}\left[\left(2 \theta+f_{2 \theta}\right) / 2\right]}
$$

A Figura 3b mostra o comportamento do teor de carbono na austenita retida em função da taxa de resfriamento para a amostra testada. É observado que o teor de carbono cresce com o aumento da taxa de resfriamento, com comportamento similar ao da Figura 3 a.

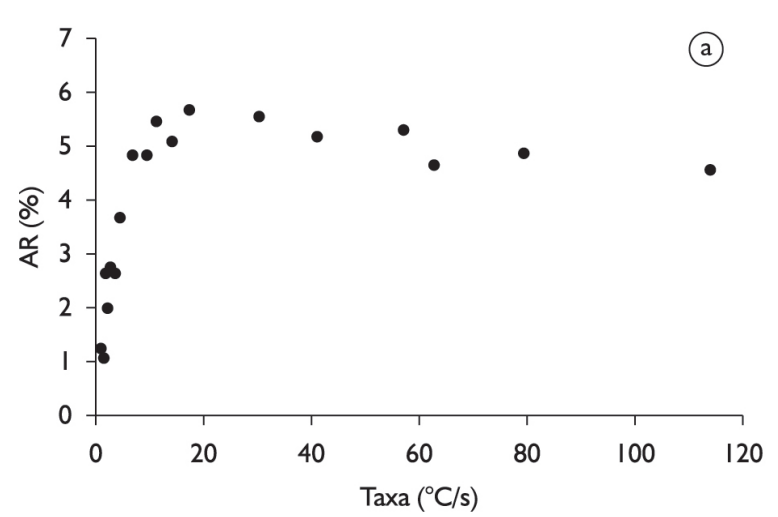

\subsection{Forma do Pico (200) $\alpha$}

Foi observado que o parâmetro $K$ da equação de Pearson VII, referente à radiação $\lambda_{\text {Kal }}$, apresenta um comportamento característico em função da presença da fase majoritária. Sendo assim, para uma taxa de resfriamento baixa, onde a ferrita é a fase em maior fração volumétrica, - valor deste parâmetro $K$ apresenta um valor elevado. Este parâmetro tem seu valor diminuído com a presença de bainita e apresenta os menores valores para taxas de resfriamento elevadas, onde a fase majoritária é a martensita. O comportamento deste parâmetro é ilustrado na Figura 4 , onde são mostradas, também, as microestruturas características para cada região e o aspecto do pico (200) $\alpha$ para dois valores extremos de $K$.

A Figura 5 mostra as medidas do \% de austenita retida em função da taxa de resfriamento, resultados da Figura 3a, porém com o eixo da abcissa em escala logarítmica invertida, da maneira que é usual nos diagramas TRC. A taxa para ocorrência máxima de austenita retida corresponde a $16,6^{\circ} \mathrm{C} / \mathrm{s}\left(1.000^{\circ} \mathrm{C} / \mathrm{min}\right)$. A taxa para ocorrência mínima de austenita retida, extrapolação para $A R(\%)=z e r o$ na Figura 5 , é de $0,8^{\circ} \mathrm{C} / \mathrm{s}\left(50^{\circ} \mathrm{C} / \mathrm{min}\right)$. Estes valores estão em coerência com a previsão do parâmetro $K$, conforme mostrado na Figura 4.

\section{DISCUSSÃO}

Os resultados mostraram que a presença de austenita retida se inicia na taxa de $0,8^{\circ} \mathrm{C} / \mathrm{s}$ e tem sua fração volumétrica crescente com da taxa de resfriamento. Para taxa próxima de $16,6^{\circ} \mathrm{C} / \mathrm{s}$ a austenita retida passa por um máximo e ficando estabilizada, ou ligeiramente decrescente, para taxas superiores.

Em relação à microestrutura, existem três regiões de predominância das fases. Para a região de baixas taxas (inferior a $16,6^{\circ} \mathrm{C} / \mathrm{s}$, mas acima de $0,8^{\circ} \mathrm{C} / \mathrm{s}$ ), a fase predominante é ferrita com presença de perlita e bainita. Para a região intermediária, com taxas entre $16,6^{\circ} \mathrm{C} / \mathrm{s}$ e

Figura 3. (a) Fração volumétrica da austenita retida (AR) em função da taxa de resfriamento; e (b) teor de carbono da austenita retida como função desta taxa. 

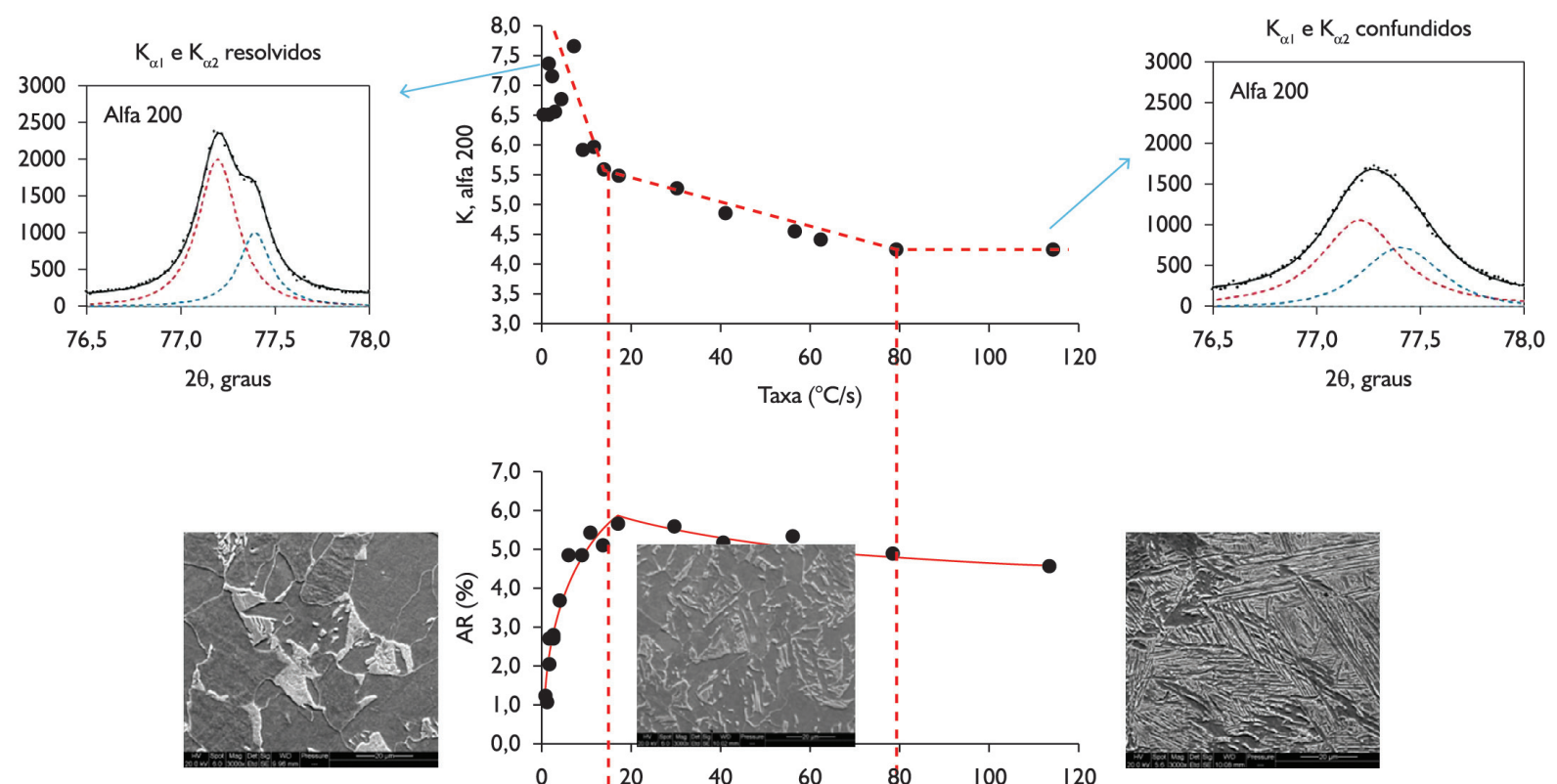

Ferrita + perlita,

Bainita surgindo

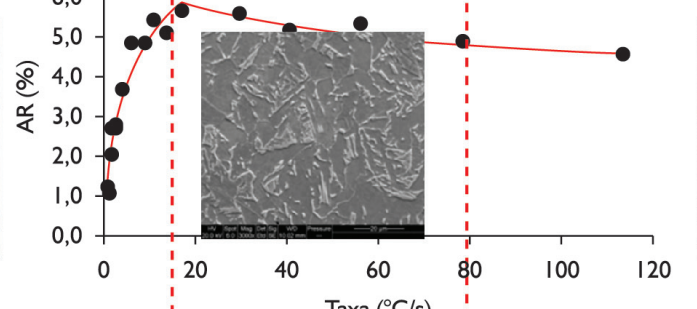

Martenista,

Ferrita + Bainita,

Martensita surgindo

Cessa formação de bainita

Figura 4. Variação do parâmetro $K$ da função de Pearson VII do pico (200) $\alpha$ com a taxa de resfriamento e comparação com o teor de austenita retida. As micrografias de MEV (ataque com Nital 2\%, aumento de 3.000X no original), mostram as fases majoritárias em cada uma das três regiões principais. Os dois gráficos menores mostram como a forma do pico (200) $\alpha$ é afetada pela taxa de resfriamento.

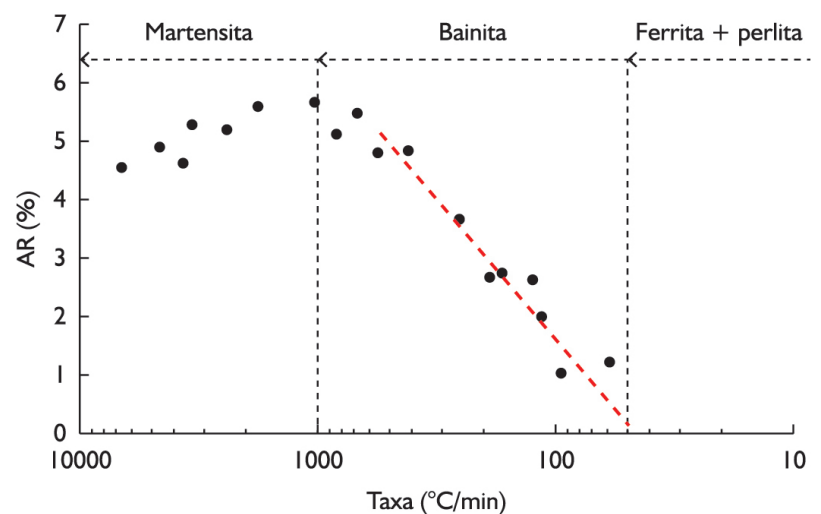

Figura 5. Fração volumétrica da austenita retida em função da taxa de resfriamento no ensaio Jominy. Escala logarítmica invertida na abcissa. Início de formação de bainita, e de austenita retida, em torno de $50^{\circ} \mathrm{C} / \mathrm{min}$. Início de formação martensítica em torno de $1.000^{\circ} \mathrm{C} / \mathrm{min}$.

$80^{\circ} \mathrm{C} / \mathrm{s}$ a fase predominante é bainita com presença crescente de martensita. Finalmente para taxas superiores a $80^{\circ} \mathrm{C} / \mathrm{s}$ a fase predominante é martensita.

A ocorrência de um máximo de austenita retida pode estar associada ao teor de carbono da austenita. A austenita tem o teor de carbono aumentado devido ao aumento gradativo de bainita. Com o surgimento da martensita a partir de $16,6^{\circ} \mathrm{C} / \mathrm{s}$, o teor de carbono na austenita retida é gradativamente reduzido até o completo desaparecimento da bainita. A partir da taxa de $80^{\circ} \mathrm{C} / \mathrm{s}$, a austenita apresenta um teor de carbono mais baixo e, portanto menos estável, pois a martensita formada tem composição da fase "mãe" restando assim, menos carbono presente na austenita.

O surgimento das fases majoritárias ferrita, bainita e martensita pode ser acompanhado através do comportamento do parâmetro $K$ da função de Pearson VII, do pico (200) $\alpha$ referente ao comprimento de onda $\lambda_{\text {Kal. }}$ Para ferrita, 0 valor deste parâmetro apresenta um valor mais elevado em relação aos valores apresentados quando a estrutura contém a presença de martensita/bainita. Assim existe separação clara entre os picos referentes aos comprimentos de onda $\lambda_{\mathrm{K} \alpha \mathrm{l}}$ e $\lambda_{\mathrm{K} \alpha 2 \text {, }}$ quando a estrutura é majoritariamente ferrítica, mas estes picos aparecem confundidos quando a estrutura é majoritariamente martensítica.

Para ausência de austenita retida, e outras fases duras associadas, é necessária uma taxa de resfriamento inferior a $0,8^{\circ} \mathrm{C} / \mathrm{s}$, guiando assim o projeto do ciclo de resfriamento do fio máquina no processo Stelmor ${ }^{\circledR}$, em aço a ser usado para eletrodos de solda. Desta forma, foi possível criar, também, uma alternativa para tratamento do fio máquina, antes da trefilação, com a implantação de ciclo mais rápido em substituição ao ciclo anteriormente utilizado (recozimento intercrítico), com os ganhos de tempo e energia associados a esta simplificação.

A proporcionalidade da austenita retida com o surgimento de fases duras gera, ainda, a possibilidade de se usar a difração de raios- $X$ como exame liberatório de fio máquina deste tipo de aço destinado a trefilação direta. 


\section{CONCLUSÕES}

A combinação da técnica de seccionamento de corpo de prova Jominy com medição de espectros de difração de raios-X, permitiu a avaliação quantitativa da presença de austenita retida em aço para eletrodo. A partir desses dados pode-se concluir que a fração volumétrica da austenita varia com a taxa de resfriamento e apresenta um máximo para $16,5^{\circ} \mathrm{C} / \mathrm{s}$.

Os espectros de difração de raios- $X$ foram optimizados através do método dos mínimos quadrados de função não linear, com modelagem através da função Pearson VII e utilização apenas das raias $K \alpha$ referente ao comprimento de onda $\lambda_{\text {Kal }}$, o que gerou melhor precisão nas medidas de austenita retida.

Através da avaliação do parâmetro $K$ da função Pearson VII, modelando o pico (200) $\alpha$, referente ao comprimento de onda $\lambda_{\text {Kal }}$, é possível prever a fase majoritária presente.

O teor de carbono na austenita retida segue o comportamento da fração volumétrica, aumentando com a taxa de resfriamento e saturando, ou diminuindo discretamente, com o surgimento de martensita.

Para que ocorra ausência de austenita retida e de outras fases duras associadas, é necessária uma taxa de resfriamento inferior a $0,8^{\circ} \mathrm{C} / \mathrm{s}$, guiando assim o projeto do ciclo de resfriamento no Stelmor ${ }^{\circledR}$, quando se procede à laminação de aço para ser usado na trefilação de arames de eletrodos de solda. Com isto houve ganho significativo através da simplificação e redução de custo de tratamento térmico posterior no cliente.

O conhecimento da proporcionalidade da austenita retida com a presença de fases duras passa a permitir o uso da difração de raios-X como exame liberatório de fio máquina neste tipo de aço.

\section{Agradecimentos}

Os autores agradecem a ArcelorMittal Monlevade pelo suporte a este projeto assim como a CAPEX/PROEX pelo apoio financeiro.

\section{REFERÊNCIAS}

I Timokhina IB, Hodgson PD, Pereloma EV. Effect of microstructure on the stability of retained austenite in transformation-induced-plasticity steels. Metallurgical and Materials Transactions. A, Physical Metallurgy and Materials Science. 2004;35(8):233I-234I. http://dx.doi.org/I0.1007/s I I66I-006-02 I3-9.

2 Kandpal BC, Chutani A, Harsimran AG, Sadanna C. A review on Jominy test and determination of effect of alloying elements on hardenability of steel using Jominy end quench test. International Journal of Advances in Engineering \& Technology. 20I I; I (3):65-7I.

3 Cullity BD, Stock SR. Elements of X-ray diffraction. 3. ed. New Jersey: Prentice Hall; 200 I.

4 Michette AG, Pfauntsch SJ. Laser plasma x-ray line spectra fitted using the Pearson VII function. Journal of Applied Physics D: Applied Physics. 2000;33(I0): I I86-I 190. http://dx.doi.org/ I0. 1088/0022-3727/33/I0/308.

5 Dmitrieva O, Ponge D, Inden G, Millan J, Choi P, Sietsma J, et al. Chemical gradients across phase boundaries between martensite and austenite in steel studied by atom probe tomography and simulation. Acta Materialia. 201 I;59(I):364-374. http://dx.doi.org/10.1016/j.actamat.2010.09.042.

6 American Society for Testing and Materials. ASTM A255-2: Standard test methods for determining hardenability of steel. West Conshohocken: ASTM; 2002.

7 American Society for Testing and Materials. ASTM E975-95: X-ray determination of retained austenite in steel with near randon crystallographic orientation. Philadelphia: ASTM; 1995.

8 Billo EJ. Excel for scientists and engineers. New York: John Wiley and Sons; 2007. Nonlinear regression using the solver; p. 313-339. http://dx.doi.org/I0.1002/97804701267/4.chI4.

9 Roberts CS. Effect of carbon on the volume fraction and lattice parameters of retained austenite and martensite. Journal of Metals Transactions AIME. 1953;197:203-204.

Recebido em: 14 Set. 2014

Aceito em: 29 Jan. 2015 